\title{
MEASURING AEROSOL OPTICAL DEPTH (AOD) AND AEROSOL PROFILES SIMULTANEOUSLY WITH A CAMERA LIDAR
}

\author{
John Barnes ${ }^{1 *}$, Robert Pipes ${ }^{1}$, Nimmi C. P. Sharma ${ }^{2}$ \\ ${ }^{I}$ NOAA/Mauna Loa Observatory, Hilo, Hawaii, USA, *Email: john.e.barness@noaa.gov \\ ${ }^{2}$ Department of Physics and Engineering Physics, Central Connecticut State University, New Britain, CT
}

\begin{abstract}
CLidar or camera lidar is a simple, inexpensive technique to measure nighttime tropospheric aerosol profiles. Stars in the raw data images used in the CLidar analysis can also be used to calculate aerosol optical depth simultaneously. A single star can be used with the Langley method or multiple star pairs can be used to reduce the error. The estimated error from data taken under clear sky conditions at Mauna Loa Observatory is approximately $+/-0.01$.
\end{abstract}

\section{INTRODUCTION}

The simplest atmospheric aerosol lidar measurement is 180 degree backscattered light. To convert this quantity into the more useful aerosol extinction or total scatter $\left(\mathrm{m}^{-1}\right)$ the extinction to backscatter ratio, also known as the lidar ratio, must be assumed or measured. If the aerosol optical depth (AOD) is known, the backscatter profile can be integrated to get an average lidar ratio.

For example, this approach is used by MPLNET [2] by using daytime AOD measured by AERONET [1]. But sun-photometer measurements of AOD are limited to daytime, when many lidars cannot operate. Starphotometers have been used to get nighttime AOD $[3,4]$ by observing single stars with a small telescope, and multiple stars have been measured simultaneously with a CCD camera [5]. But both types of photometers require an additional instrument to the lidar being used, and there can be differences in the time, location, and wavelength between the lidar and the photometer. In addition, observing single stars involves accurate pointing of the telescope, which not needed when using a wide-angle lens and camera.

This study examines the feasibility of using a novel technique of aerosol profiling, camera lidar or CLidar, to also measure AOD with no additional equipment.

\section{METHODOLOGY}

The CLidar technique uses a wide-angle lens on a CCD camera to image a laser beam, usually pointed vertically, from the ground to the zenith $[6,7,8]$. The geometry is shown in figure 1 . The technique is currently limited to nighttime conditions. A continuous laser can be used since the altitude information is determined by the distance from the camera to the laser beam (usually a few $100 \mathrm{~m}$ ), and the angle of observation. The CLidar has no overlap function as in lidar, and there is no data acquisition electronics other than the computer to operate the camera. Another difference with lidar is that light is scattered at 90 degrees at the ground and approaches 180 degree scatter as altitude increases. An important property of the fisheye lens used is that each pixel maps to a constant angle.

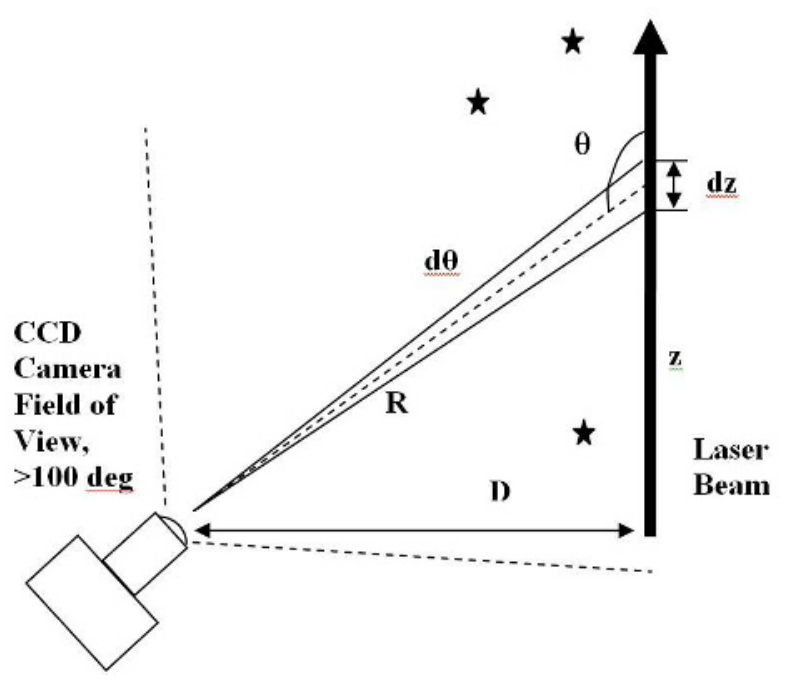

Figure 1, The geometry used for the CLidar technique along with three stars. The star brightness is a measured in the same images used for the CLidar aerosol profiles.

The integration period for the CLidar image is usually a few minutes, during which time brighter stars are also imaged. So the brightness for each 
star is being measured at the same location, time, and through the same interference filter as the laser beam. Depending on the camera resolution and the lens used, a star is usually imaged onto several pixels. Pixel counts must be checked to insure there are no errors due to saturation effects.

The standard calibration method for sunphotometers, the Langley calibration, is to measure the sun as it rises or sets during which the air mass changes. An air mass change of 2 to 8 or more is often desired. The change of the log of the star brightness with air mass is linear, and the slope and zero air mass intercept are the calibration constants. A single star can be used in the same manner, although the signal will be much weaker.

The multi star method takes advantage of star brightness measured by astronomers. By using a pair of stars and the stellar magnitudes for each, the AOD can be measured without the calibration constants. This of course is not possible for sunphotometers. In this study, brighter stars in the CLidar image were identified and the Yale Bright Star Catalog Fifth edition was used for their magnitudes and relative positions to get their air masses. Interpolations were made since the magnitude data does not match the laser wavelength. The images were analyzed using software written in IDL (Interactive Data Language). The stars signals were calculated by using an IDL 2-D Gaussian plus constant nonlinear least squares fit. Error estimates were made using the pixel counts for each star in the images, which were used with estimates of the other quantities needed in the error propagation calculation.

\section{RESULTS}

The CLidar system used a Santa Barbara Instruments Group ST-1603 CCD camera cooled to $-20 \mathrm{C}$. The rectangular chip array is 1530 by 1020, 9 micron pixels. The lens was a Sigma fisheye, $10 \mathrm{~mm}$ focal length, $\mathrm{F} / 2.8$. The laser imaged is part of the NOAA/Mauna Loa Observatory lidar, which uses a doubled Nd:YAG laser operating at about 15 Watts $(532 \mathrm{~nm})$. Aerosol profiles were acquired but only the starphotometer data are presented here.
In figure 2 an example is shown of a Langley plot of the star Rigel in the constellation Orion on 2010/11/10. As the star rose above the horizon the integration time was 1 minute. This changed to 3 minutes as the air mass decreased and wasn't changing as rapidly. A $532 \mathrm{~nm}$ interference filter with a 10 FWHH bandpass was inserted between the camera and the Sigma lens. The $\mathrm{R}^{2}$ of 0.9944 is equivalent to an AOD error of about $+/-0.02$, similar to other star-photometers $[3,4]$. There was very little aerosol over the observatory at the time, so the slope represents very clean air.

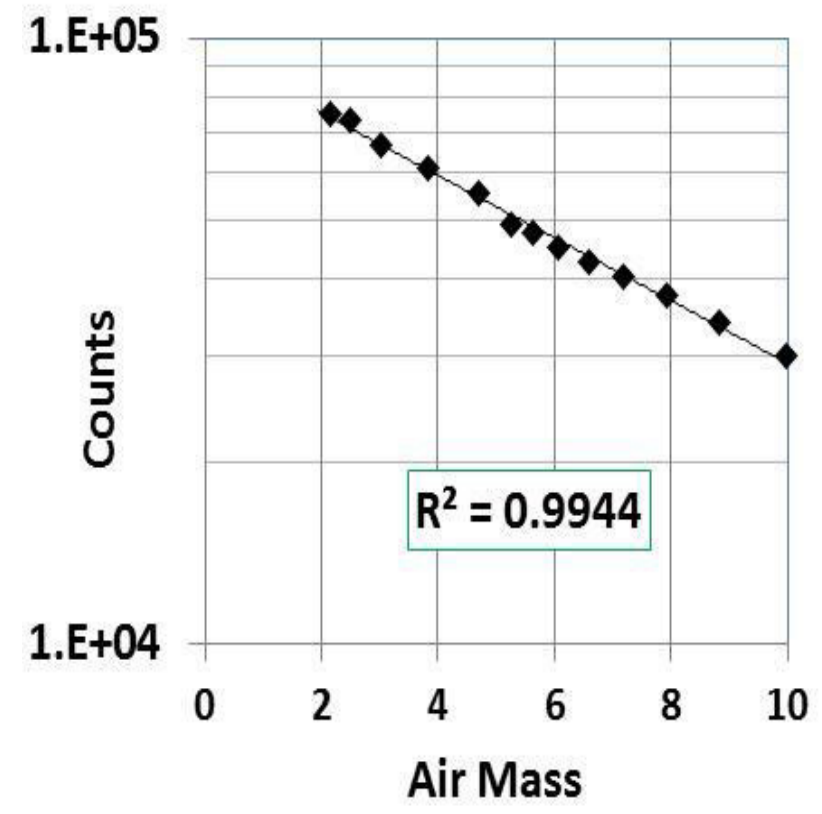

Figure 2, Langley plot using the star Rigel in Orion under very low aerosol conditions. The $\mathrm{R}^{2}$ of 0.9944 is equivalent to an AOD error of about 0.02 .

The multi-star method was also tested at Mauna Loa Observatory under very clean aerosol conditions on 2014/11/20. The exposure time was 15 seconds. When using a pair of stars to calculate AOD, the signal for each star is compared to its stellar magnitude. To minimize the error it is best to choose stars with different air masses. The best results use a star low in the sky (high air mass, high extinction) and another higher in the sky (low air mass, low extinction). In this example 10 of the brighter stars in the image were chosen which created 45 possible pairs. Many of these pairs used stars with similar air masses and generated large errors. 
Figure 3 shows the results for the 5 star pairs which had a calculated AOD error of less than +/0.05 . The AODs measured by AERONET on the daytime before and the daytime after this night were about 0.006 . This should be an upper limit on the real AOD on this night since the air generally gets cleaner at night at Mauna Loa Observatory. There is currently a constant offset of -0.079 AOD generated in the analysis. This offset has been subtracted from the results shown. The source of the offset is currently under investigation, but it is not believed that it affects the AOD error estimates quoted here.

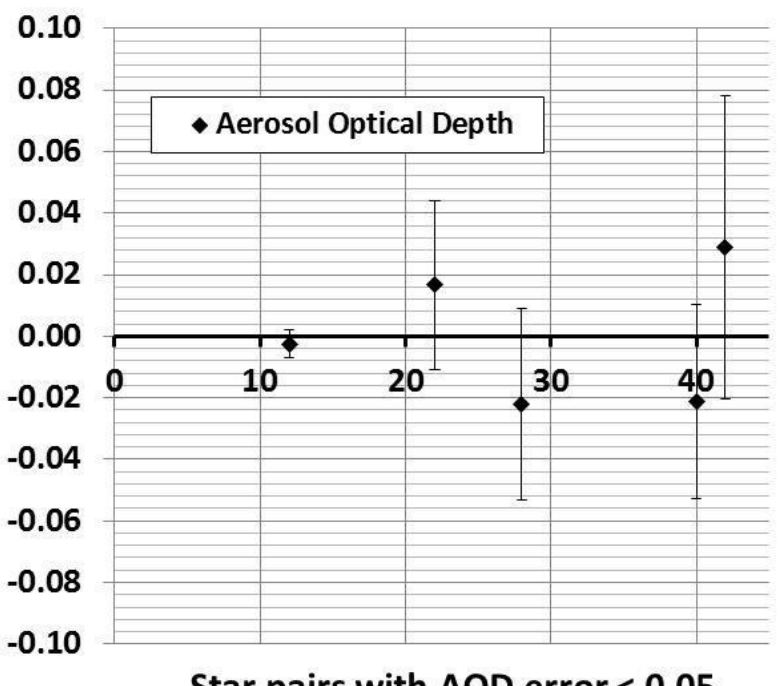

Star pairs with AOD error $<0.05$

Figure 3, AOD measurements of clean sky conditions at Mauna Loa Observatory on 2014/11/10 with a 15 second exposure. The best 5 out of 45 pairs are shown.

Using all five star pairs results in an AOD error of $+/-0.0042$. In figure 3 a single star pair, \#12, is seen to dominate the overall error. It has a calculated error $+/-0.0044$. Without this pair the error is $+/-0.018$. There are other possible factors in the error calculation that may not be included such as the uniformity of the sensitivity of the CCD chip. These are currently under investigation but it is believed the error will lie between the 0.0044 and 0.018 values. The achievable error will depend on the actual operating conditions such as the number of bright stars available in the image, and background light at the observing location.

\section{CONCLUSIONS}

The simplicity and low cost of the CLidar technique is an attractive characteristic when measuring nighttime tropospheric aerosols. The knowledge of AOD is a strong constraint on converting the aerosol backscatter profile to an aerosol extinction profile. Using star brightness in the CLidar images to measure AOD is a promising addition with an error of +/- 0.01 possible.

\section{ACKNOWLEDGEMENT}

This work is supported by the National Oceanic and Atmospheric Administration/Earth System Research Laboratory/Global Monitoring Division.

\section{REFERENCES}

\section{[1] http://aeronet.gsfc.nasa.gov/}

[2] http://mplnet.gsfc.nasa.gov/

[3] Leiterer, U., et al., 1995: A new star photometer developed for spectral aerosol optical thickness measurements in Lindenberg, Beitr. Phys. Atmosph., 68, 133-141.

[4] Perez-Ramirez, D. et al., Development and calibration of a star photometer to measure the aerosol optical depth: smoke observations at a high mountain site, Atmos. Environ., 42, 27332738 ..

[5] Lanciano, O. and G. Fiocco, 2007: Nighttime measurements of atmospheric optical thickness by star photometery with a digital camera, Applied Optics, 46, 5176-5182.

[6] Barnes, John E., S. Bronner, Robert Beck, and N. C. Parikh, 2003: Boundary Layer Scattering Measurements with a Charge-Coupled Device Camera Lidar, Applied Optics, 42, no. 15, 2647-2652.

[7] Barnes, John E., N.C. Parikh Sharma and Trevor Kaplan, Atmospheric aerosol profiling with a bistatic imaging lidar system, 2007: Applied Optics, 46, no. 15, 2922-2929.

[8] Nimmi C. P. Sharma and John E. Barnes, 2015: CLidar mountain boundary layer case studies, $27^{\text {th }} I L R C$, New York City. 